\title{
Microwave Components with MEMS Switches
}

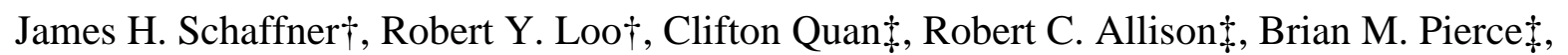 \\ Stanley W. Livingston $\neq$, Adele E. Schmitz $\dagger$, Tsung-Yuan Hsu†, Daniel F. Sievenpiper†, Frank \\ A. Dolezal $\dagger$, Gregory, L. Tangonan $\dagger$ \\ $\dagger$ HRL Laboratories, LLC, Malibu, California, USA, \\ \$ Raytheon Systems Company, El Segundo, California, USA \\ jhschaffner@HRL.com
}

\begin{abstract}
RF MEMS switches with metal-metal contacts are being developed for microwave applications where broadband, high linearity performance is required. These switches provide less than $0.2 \mathrm{~dB}$ insertion loss through $40 \mathrm{GHz}$. This paper describes the integration of these switches into selected microwave components such as reconfigurable antenna elements, tunable filters, switched delay lines, and SPDT switches. Microwave and millimeter wave measured results from these circuits are presented.
\end{abstract}

\section{INTRODUCTION}

When low insertion loss and high linearity are required over a broad frequency band, mechanical switches are better than solid-state switches. Attention is currently focused on the development of RF microelectro-mechanical system (MEMS) switches. While issues of reliability, robustness, and performance of these switches are of paramount importance, it is also essential that these switches be demonstrated in prototype components to fuel interest for system designers. Some components with RF MEMS switches have already been reported, for example, microwave and millimeter wave phase-shifters [1]. In this paper, the performance of a few microwave components that have been integrated with the RF MEMS switches that are being developed at HRL Laboratories will be described. These MEMS switches have been inserted and demonstrated in reconfigurable antenna elements, tunable filters, switched delay lines, and single-pole double-throw (SPDT) switches.

\section{METAL CONTACT RF MEMS SWITCHES}

The switch, shown in figure 1 , is a microscopic version of a conventional relay cantilever switch [2] that is fabricated on top of a GaAs substrate. The RF portion of the switch has a series gap in microstrip transmission line that can be short circuited by a bar of gold deposited at the end of a cantilever beam. Electrostatic actuation is used to pull a cantilever beam down to close the switch. The cantilever beam is made of three layers (silicon nitride/gold/silicon nitride) in order to compensate for mechanical stress so that the beam remains flat without bending when the switch is not actuated. A pure silicon nitride section in the cantilever arm between the top actuation electrode and the metal shorting bar forms a DC block and also reduces coupling from the RF line into the DC bias circuitry. As can be seen in figure 1 , the switch is comprised of two springs, one that presses the shorting bar down across the gap under actuation, and the other near the cantilever anchor point that provides restoring force to lift the cantilever up after the actuation voltage is removed.

Low RF contact resistance $(<1 \Omega)$ is achieved by two metal dimples located at the edges of the shorting bar which increase the contact force when the beam is in the closed position. The microwave performance of the series switch is shown in figure 2. Typical measured parameters of these switches is very low insertion loss, less than $0.2 \mathrm{~dB}$ up to $40 \mathrm{GHz}$; and high isolation, greater than $40 \mathrm{~dB}$ at frequencies less than $5 \mathrm{GHz}$ which slowly decreases to about $25 \mathrm{~dB}$ at $40 \mathrm{GHz}$. These switches have also been tested with up to one watt of RF power when hot switched and up to two watts of RF power when cold switched, without any resulting degradation in performance.

\section{MEMS SWITCHES IN MICROWAVE COMPONENTS.}

Some microwave circuit components have been built which integrate the RF MEMS switches described in the previous section. In this section, RF MEMS based reconfigurable antenna elements, tunable filters, truetime delay phase shifters, and hybrid SPDT switches will be described.

First, an essential element of any microwave wireless system is the antenna. RF MEMS switches enable antennas to be reconfigured for both frequency agility and for beam steering. As an example, MEMS switches were used in a microwave dipole to tune the resonant length of the dipole for operation at X-band and Ku-band. The printed circuit dipole, shown in figure 3, was fed by a slot coupled microstrip line in a multi-layer alumina substrate. The measured patterns are also presented in figure 3. When the switches were closed, the dipole was resonant at $11 \mathrm{GHz}$. When the switches 
were opened, effectively shortening the dipole length, the resonant frequency increased to $18 \mathrm{GHz}$. No backside groundplane was present during these measurements; hence, the back-scattered radiation is apparent in these figures. In addition to the dipole, RF MEMS switched slot and notch reconfigurable antenna elements are being investigated.

Another microwave circuit that was fabricated and tested was a MEMS tunable notch filter. This filter was created by placing three equally spaced open circuited microstrip stubs along a microstrip transmission line. MEMS switches were inserted into gaps in the stubs and then used to change the effective length of the stubs. In one design, the notch frequency changed from 18 to $32 \mathrm{GHz}$, when the switches went from closed to open. The application of these switches for tunable bandpass filters requires careful consideration, since even a loss per switch of $0.1-0.2 \mathrm{~dB}$ can have deleterious consequences to the $\mathrm{Q}$ of the filter elements. However, the use of RF MEMS switches for switched filter banks is very promising for low-loss microwave receivers.

The low loss and high isolation of metal contact RF MEMS switches is ideal for microwave switched delay lines for phase shifters. Figure 4 shows the result from an RF MEMS switched delay line one-bit phase shifter where the difference between the delay arm and the reference arm was designed to be $180^{\circ}$ at $10 \mathrm{GHz}$. This phase shifter was fabricated as a hybrid structure with a ceramic microstrip delay line and discrete RF MEMS phase shifters. The difference in the insertion loss between the two arms was due to transmission line losses and not the switches. The shift in the expected frequency response was caused by parasitic circuit elements such as bond wires and discrete chip effects.

Finally, a hybrid SPDT is shown in figure 5. Here, two microstrip metal-metal contacts are wirebonded together along with $\mathrm{J}$-micro ${ }^{\circledR}$ coplanar-tomicrostrip adapters. The results from this measurement are shown in figure 6.

\section{CONCLUSION}

In conclusion, RF MEMS switches have been demonstrated in a number of basic microwave components. While much work is still needed to insure the reliability of these switches, the circuits and measurements presented here should help to instill confidence in the future of the MEMS switch as a standard microwave device.

\section{ACKNOWLEDGMENT}

This work was supported in part by the DARPA MAFET-III program, contract \#N66001-96-C-8636.

\section{REFERENCES}

[1]. B. Pillans, S. Eshelman, A. Malczewski, J. Ehmke, and C. Goldsmith, "Ka-band RF MEMS phase shifters," IEEE Microwave and Guided Wave Letters, vol. 9, no. 12, December 1999, pp. 520-522.

[2]. D. Hyman, A. Schmitz, B. Warneke, T. Y. Hsu, J. Lam, J. Brown, J. Schaffner, A. Walston, R.Y. Loo, G. L. Tangonan, M. Mehregany, and J. Lee, "GaAs-Compatible Surface-Micromachined RF MEMS Switches," Electronn Lett., Vol. 35, No. 3, February 4, 1999, pp. 224226 .

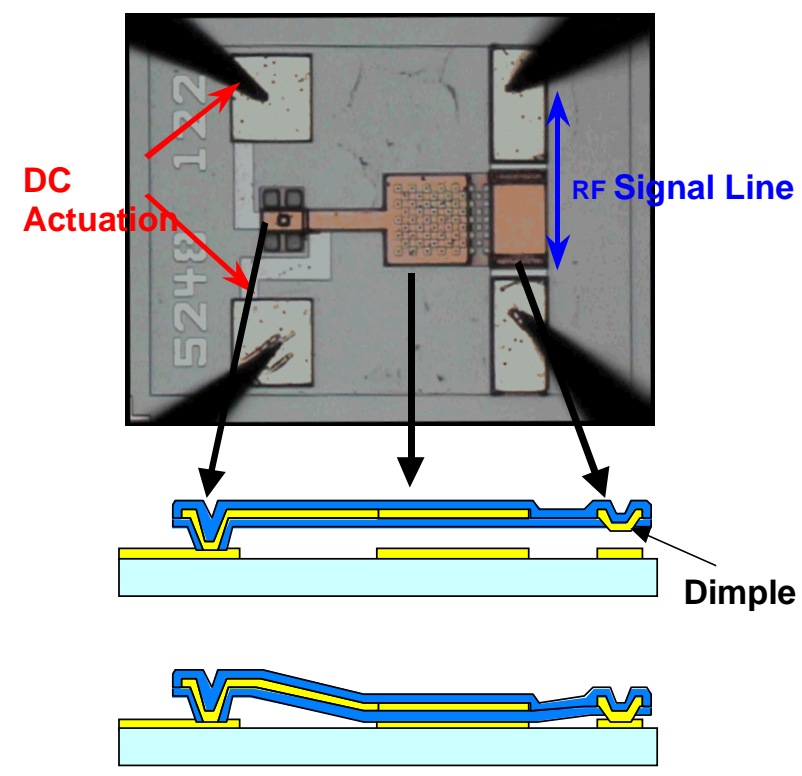

Fig. 1. A photograph of an RF MEMS switch (top view) and the cantilever beam action (side view) for both the open and closed positions.
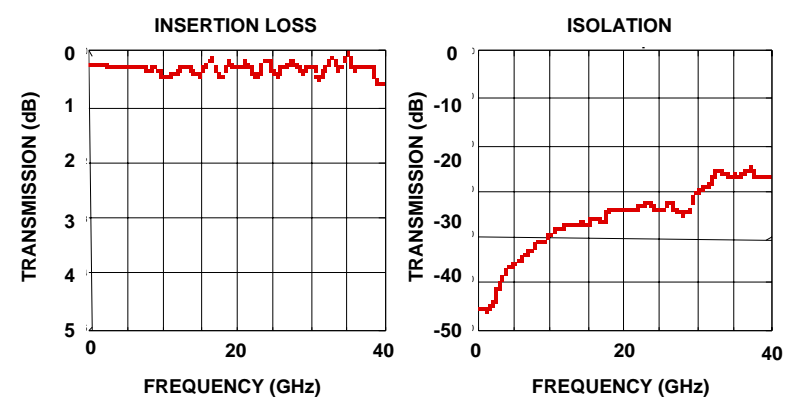

Fig. 2. Measured insertion loss of a closed switch and isolation of an open switch. 

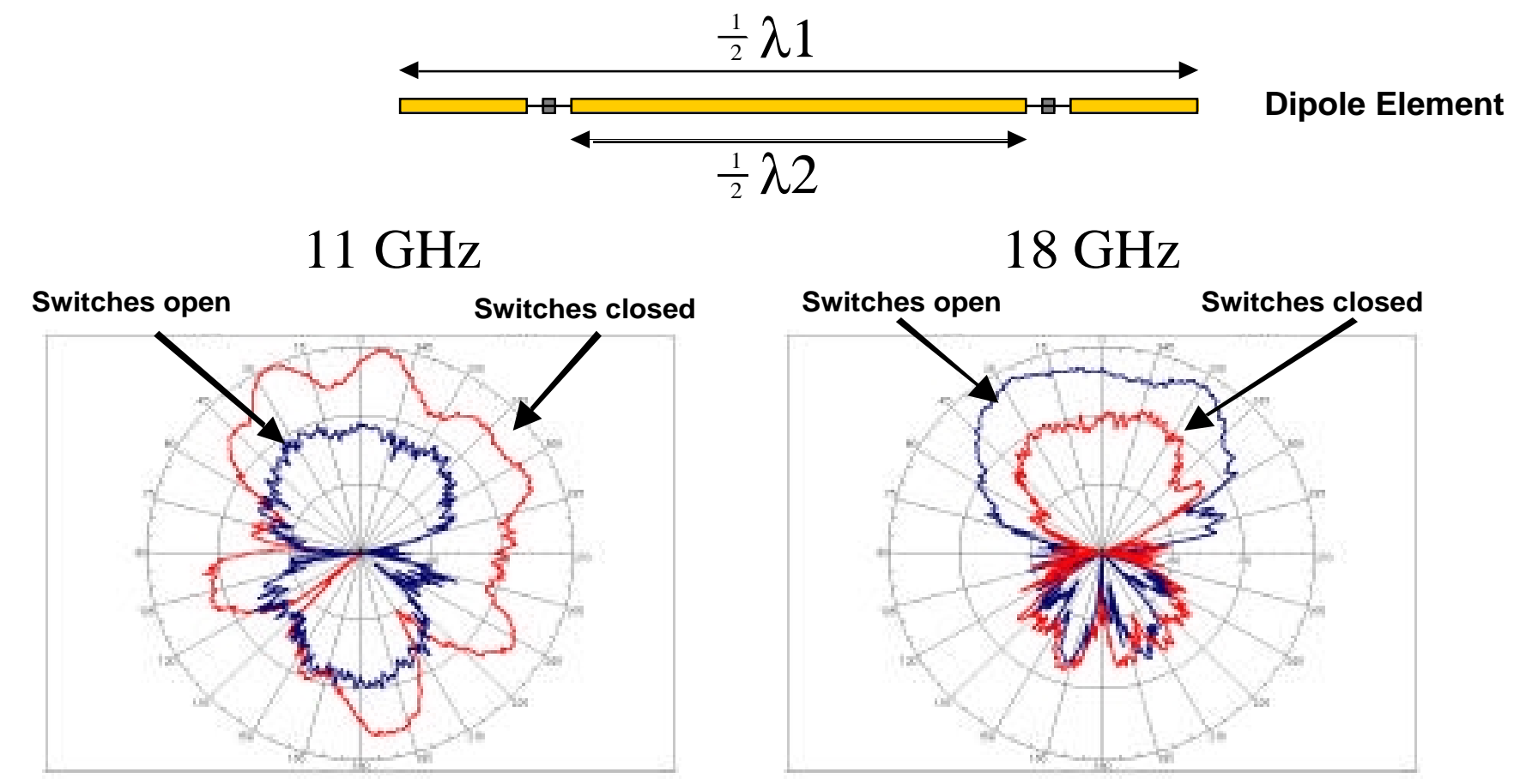

Fig. 3. Antenna patterns of the MEMS switch tunable printed circuit dipole with resonance frequencies of 11 and $18 \mathrm{GHz}$. No ground plane was used in this measurement, thus resulting in a back radiation.

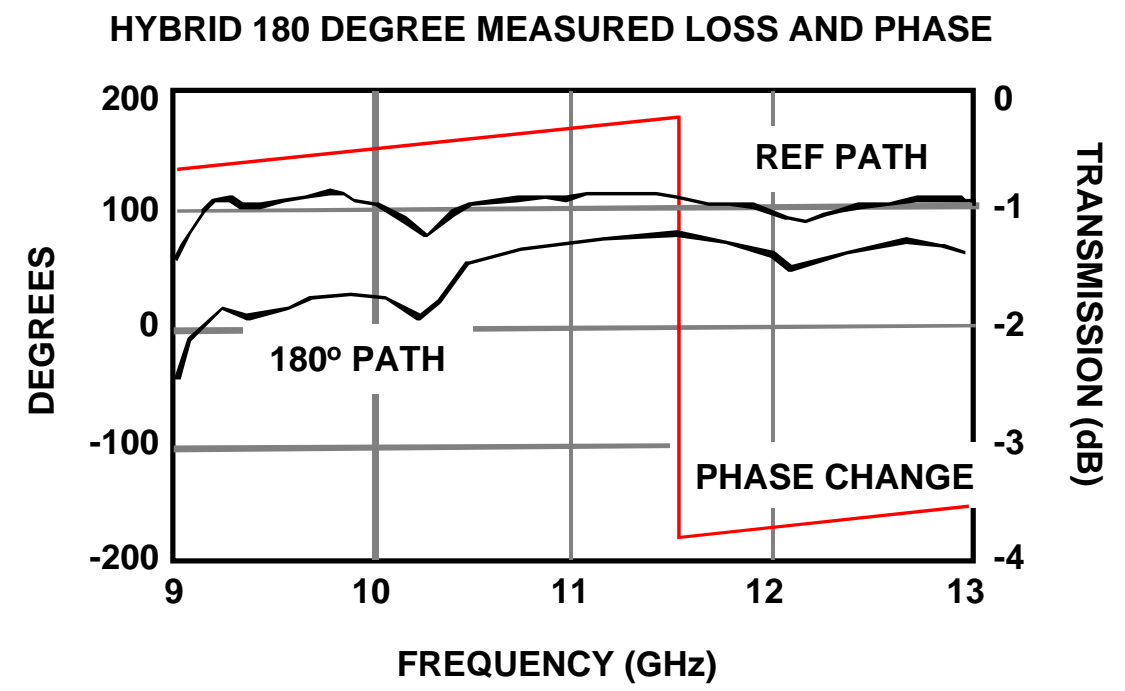

Fig. 4. Measured results from a $180^{\circ}$ delay-line phase shifter. The phase change is the difference in degrees between the reference path and the $180^{\circ}$ path. 


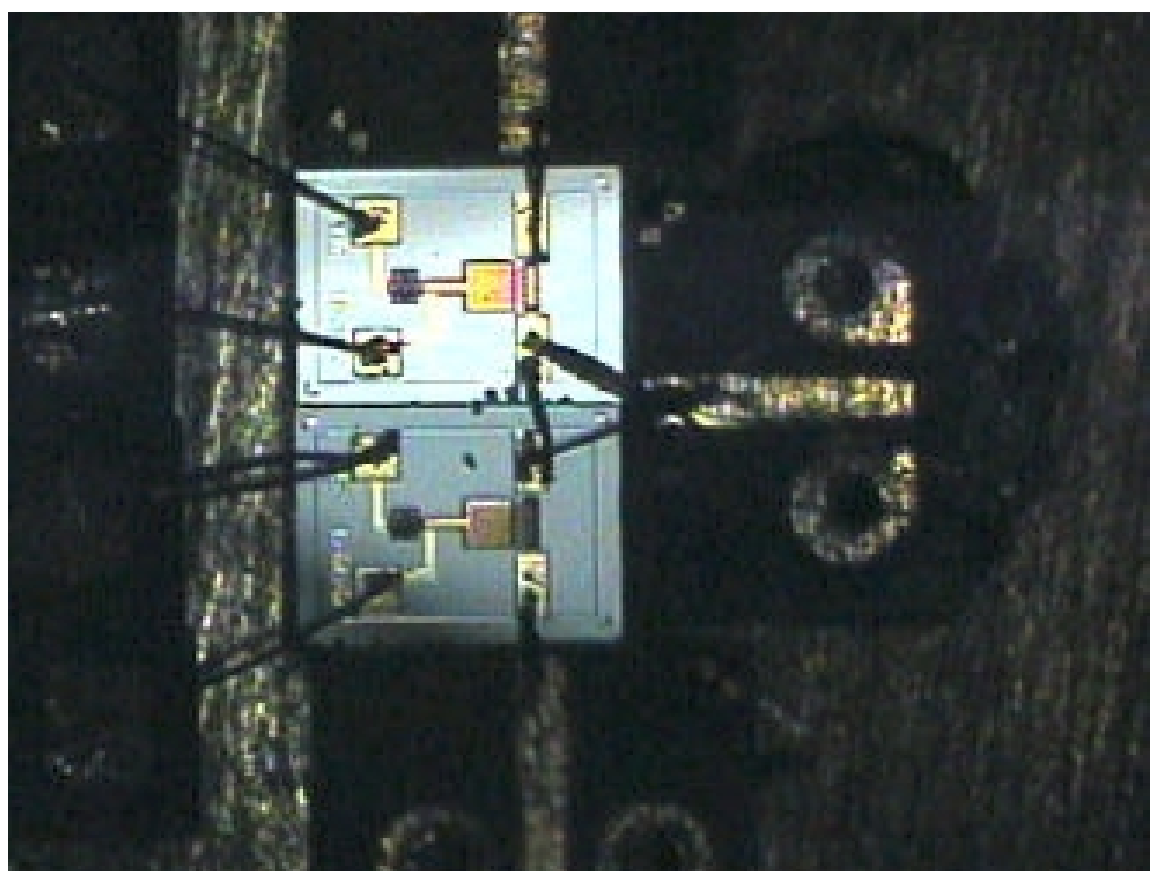

Fig. 5. A hybrid single-pole double-throw switch.
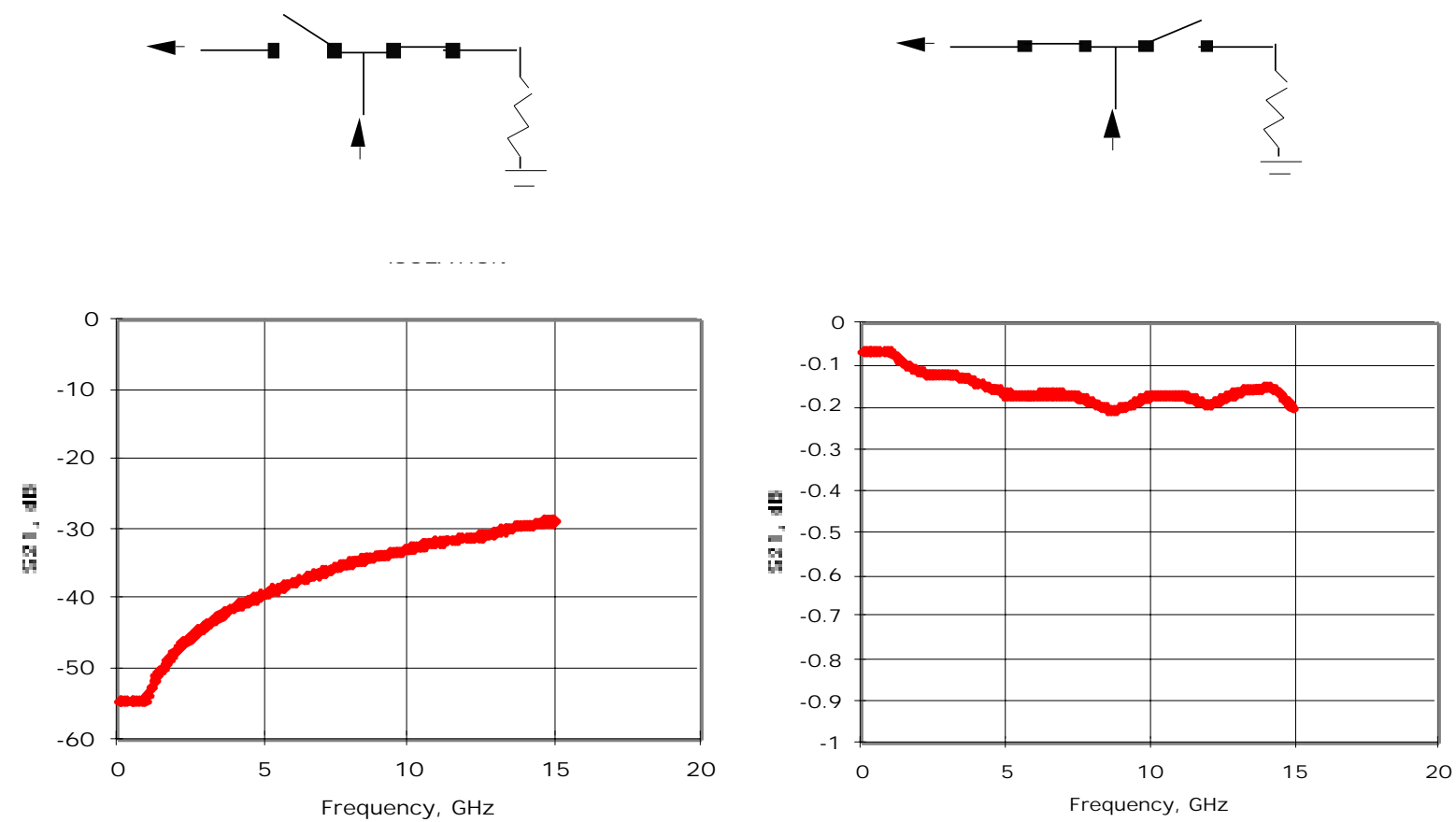

Fig. 6 Performance of the hybrid single-pole double-throw switch up to $15 \mathrm{GHz}$. 